\title{
Stress-strain synchronization for high strain rate tests on brittle composites
}

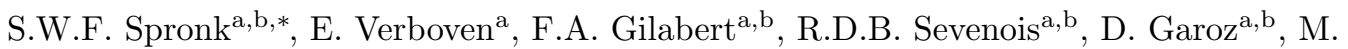 \\ Kersemans $^{\mathrm{a}}$, W. Van Paepegem ${ }^{\mathrm{a}}$ \\ ${ }^{a}$ Ghent University - Department of Materials, Textiles and Chemical Engineering (MaTCh), Faculty of \\ Engineering and Architecture, Ghent University, Tech Lane Ghent Science Park - Campus A, \\ Technologiepark-Zwijnaarde 903, B-9052 Zwijnaarde, Belgium \\ ${ }^{b}$ SIM Program M3Strength, Technologiepark-Zwijnaarde 935, B-9052 Zwijnaarde, Belgium
}

\begin{abstract}
Nowadays, many researchers develop rate-dependent composite material models for application in dynamic simulations. Ideally, full stress-strain curves at a wide range of strain rates are available for identification of the different parameters of these models. Dynamic tensile tests are needed to produce the experimental input data. However, especially for brittle materials, the data acquisition during these tests becomes critical. The effect of synchronization on the test results is investigated by conducting a series of dynamic tensile tests on three different brittle continuous-fibre composite laminates. It is demonstrated that synchronization errors of the order of 1 microsecond already have a significant effect on the test outcome at high rates. With the aid of a finite-element model, the limiting factors on the maximum attainable strain rate are quantified.
\end{abstract}

Keywords: High strain rate, Tensile behaviour, Data reduction, Digital image correlation (DIC), Polymer-matrix composites (PMCs)

\section{Introduction}

Car manufacturers attempt to reduce the mass of the next generation of vehicles by using composite materials for structural elements like door sills and B-pillars. In a vehicle crash, materials deform at rates between quasi-static and about $200 \mathrm{~s}^{-1}$ [20]. Composite behaviour is known to depend on strain rate [19] and the material properties should, therefore, be obtained under the right conditions. A test series is needed in which tests at several speeds are performed to obtain a global overview of material rate-dependency.

This current research aims at obtaining accurate stress-strain curves at every decade of strain rate between quasi-static and $200 \mathrm{~s}^{-1}$ to make a full rate-dependent parameter identification possible. The focus lies on relatively brittle composites with a strain to failure of $1 \%$ or lower, because these are found to be the most difficult to obtain high rate test data for.

\footnotetext{
* Corresponding author

Email address: Siebe.Spronk@UGent.be (S.W.F. Spronk)

URL: http://www. composites.ugent.be (S.W.F. Spronk)
} 


\subsection{Tensile rate dependence in the literature}

The most common method of investigating material behaviour is the tensile test. It is less common to perform this test at strain rates close to the aforementioned upper limit. Dynamic tensile test data for composites in literature typically does not contain values in the strain rate range of about 10 to $500 \mathrm{~s}^{-1}$. The reason for this is the following. One, stress progresses at a finite speed, invalidating the assumption of equilibrium at these deformation speeds. Measuring load and strain at different locations is then no longer possible. Two, the test duration approaches the period of the natural frequency of different parts of the test bench. Both effects mark the boundary above which a switch to bar-impact testing seems necessary while at lower speeds the standard tensile test is most effective. Close to the boundary, both test methods are difficult to apply.

Literature with complete stress-strain curves of continuous-fibre composites close to the aforementioned upper limit, say between 100 and $300 \mathrm{~s}^{-1}$, is scarce. Looking for works about composites with a typical failure strain at or below $1 \%$, only four useful references were found. Three of these belong to a test series by Daniel. He managed to obtain curves for $\mathrm{C} / \mathrm{E}$ at these strain rates by using an explosion to expand a composite ring, creating tension in the circumferential direction 24 4]. Strain was measured using strain gauges on the composite specimen. Stress was measured by expansion of an adjacent steel ring with strain gauges [4. The other record that contains full tensile stress-strain curves in the range of interest is by Kuhn et al., who used a split-Hopkinson pressure bar (SHPB) adapted to apply tension 13. They performed dynamic tension tests on unidirectional $\mathrm{C} / \mathrm{E}$ in the transverse direction. The average strain rate of the tests amounts to $271 \mathrm{~s}^{-1}$, and their full stress-strain curves go up to a strain of about $0.8 \%$. No data below that rate is given, probably caused by the limits of the chosen test method.

No test standard is available to date that deals with high-rate tensile testing of continuous-fibre composites. The only related documents available either deal with metals [6, 11] or with polymers [9, 17. It is worth mentioning that the ISO 18872 standard for polymers evades the use of high rate testing by using the results at low- and intermediate rates to estimate the behaviour at high rate by extrapolation [9]. The formulae used are optimised for pure polymers and would probably produce wrong results when applied to composites.

\subsection{Dynamic tensile test methods}

Typically dynamic tension experiments are carried out using (i) a standard test bench close to its high velocity limit, (ii) a drop-weight tower with some mechanism to convert the impact load to tension, (iii) a hydraulic pulse machine, or (iv) a SHPB set-up with a special striker mechanism to produce a tensile strain wave [19].

The expanding ring test method mentioned earlier is a relatively uncommon way to test composites in tension, and Daniel shows it can be used to test both quasi-statically and at strain rates 
close to the currently required upper limit (e.g. [4). The material is, however, provided in plate shape for the current research, so the method is not further considered here.

The hydraulic pulse test bench is the only machine that can serve for all current strain rates of interest without the need for a change in equipment or method. It applies an open-loop scheme for the higher velocities, and a slack rod allows for some unconstrained acceleration of the actuator before it pulls on the specimen at a fairly constant velocity. The use of a single test set-up for all strain rates removes the possible effect of a change of equipment on the results. The question remains whether the set-up is suitable to achieve the target rate of $200 \mathrm{~s}^{-1}$.

\subsection{Data acquisition}

The challenge of dynamic tensile testing of brittle materials is the very short test duration. The failure strain of a 90-degree-unidirectional (UD) composite is small: typically $0.6 \%$ in the case of one of the materials used for this research. Consequently, the test duration is $30 \mu \mathrm{s}$ at the maximum requested rate of $200 \mathrm{~s}^{-1}$. Such a short time period has consequences for the data acquisition.

1. Any signal conditioning or amplifying device should be capable of handling sufficiently high frequencies. The SAE recommended practise for high strain rate tensile testing of polymers advises ten times the approximate maximum signal frequency, which is determined by assuming the shortest test resembles a quarter of a sine up to the yield point [17. Taking the failure point as the yield point, the minimum test duration of $30 \mu \mathrm{s}$ results in a maximum frequency of $8.33 \mathrm{kHz}$. The SAE then advises a minimum frequency response of $83.3 \mathrm{kHz}$. ISO 26203-2 for testing metallic materials at high strain rates states that the frequency response on strain should be at least $100 \dot{\varepsilon}$, and that on force at least $1000 \dot{\varepsilon}$ [11. This amounts to $20 \mathrm{kHz}$ for strain conditioning and $200 \mathrm{kHz}$ for force.

2. Data storage should take place at sufficient samples per second. A minimum would be the Nyquist frequency, which would be at twice the maximum frequency response of the conditioning equipment. ISO 26203-2 advises four times the limit frequency of the force measurement system, which would lead to $800 \mathrm{kSamples} \mathrm{s}^{-1}$.

3. The output of multiple different measuring systems needs to be synchronised. On a hydraulic pulse machine, force is typically measured by a quartz transducer and strain using strain gauges. A 30 us test duration means that these should be synchronized to, at the very least, within a single microsecond. Typically publications about dynamic tensile testing contain no information about data stream synchronisation, nor do the standards. Two minor exceptions are found. The first is the SAE J2749, in which it is stated that the data streams may need to be reconciled because of a measurable time lag [17] but gives no mention about how this should be done or what is the origin of this lag. The second is a recent publication in which optical strain measurement was applied for dynamic tensile tests on a woven glass/vinylester composite where load and strain data were manually synchronized $[8]$. 
Several questions thus arise about tests on brittle continuous-fibre composites in dynamic tension. First, how can individual data streams be synchronized in order to obtain meaningful material data? Second, what would be the maximum strain rate attainable with a hydraulic pulse set-up and what are the limiting factors?

\section{Experimental programme}

The goal is to obtain full stress-strain curves at several strain rates between quasi-static and $200 \mathrm{~s}^{-1}$. Six strain rates are chosen: $0.002,0.02,0.2,2,20$, and $200 \mathrm{~s}^{-1}$.

\subsection{Materials}

Two composite material systems, both relevant for the automotive industry, were investigated. One was Pyrofil ${ }^{\mathrm{TM}}$ TR/360 carbon/epoxy, where the epoxy is curing modified to cure in under 5 minutes above $140^{\circ} \mathrm{C}$. Both a UD variant (TR 360E250S) and a plain weave variant (TR3110 360GMP) have a failure strain of around $1 \%$ or below and were used for the current research. The second material was Cetex TC910 E-glass/polyamide-6 composite. Only the UD variant formed part of this work because it fails at strains of around $1 \%$ in the transverse direction.

\subsection{Tensile test set-up and instrumentation}

A Instron VHS z25/20 test bench was used to carry out the experiments. The maximum load capacity of the machine is $25 \mathrm{kN}$, and it can pull at speeds of up to $20 \mathrm{~ms}^{-1}$. The piston was connected to the bottom grip via a slack rod, a $2 \mathrm{~mm}$-thick nitrile rubber damping ring was inserted to prevent excitation of higher harmonics during the high speed tests.

The data-acquisition of the test set-up is depicted in figure 1 .

Force was measured using a Kistler 9061A $200 \mathrm{kN}$ piezoelectric load cell and a Kistler 5011B Charge Amplifier. Piezoelectric cells are a typical choice for dynamic tests due to their fast response time and relatively high eigenfrequency, as opposed to the strain-gauge-based cells which usually have a limited bandwidth and a low natural frequency. The amplifier has a bandwidth of $200 \mathrm{kHz}$ [12, which should be high enough to follow the fastest expected changes in load.

Data recording was performed using a 100 MSamples $^{-1}$ measurement card in a HBM GEN5i digital oscilloscope. This recording rate, and the bandwidth of at least $26 \mathrm{MHz}$ [7], were more than sufficient to record the test data.

Strain was measured using either strain gauges (SGs) or digital image correlation (DIC), or in some cases both. General-purpose $350 \Omega$ CEA-06-250UN-350 gauges by Vishay Precision Group were used to measure strain on the rear side of a specimen, the front was speckled black on a white background using a general-purpose matte spray paint.

The strain gauge was connected to a Vishay 2100 signal conditioning amplifier set at its maximum bandwidth of $35 \mathrm{kHz}$. This falls between the two requirements found in literature and section 


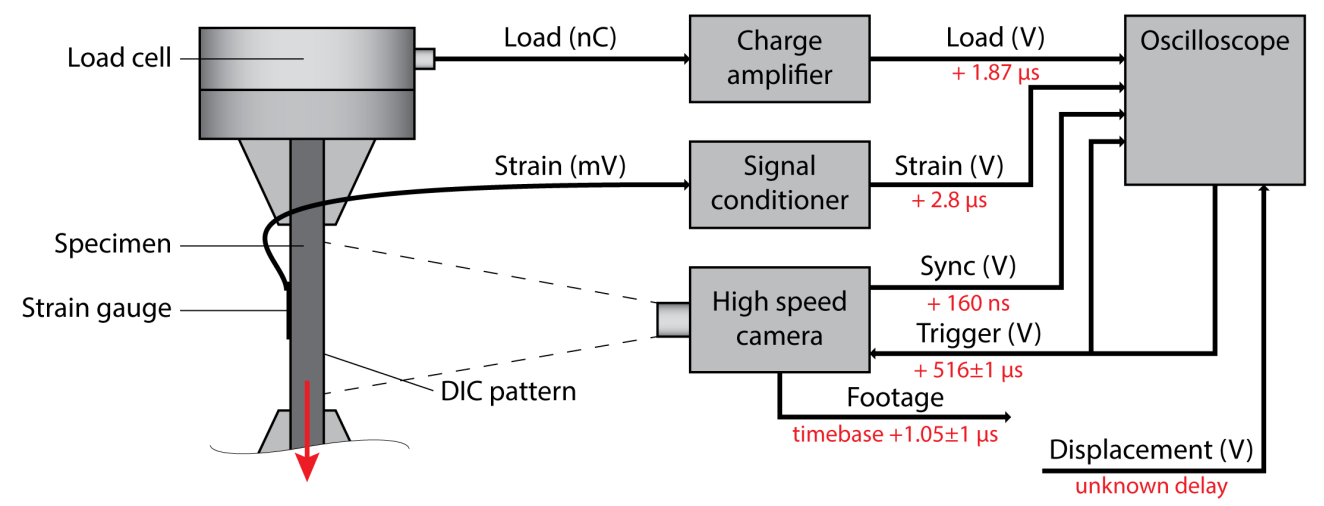

Figure 1. The data acquisition in the dynamic tension set-up. The figures in red are the non-cumulative delays of the respective signals when recording at the highest rate: for the total delay of e.g. the camera timebase, the indicated values need to be combined in the correct manner.

3.2 .2 shows that this is indeed on the low side for the current research. The digital oscilloscope records the output of the signal conditioner.

A Photron SA-4 high-speed camera (HSC) was used to capture the strain using 2D-DIC. The camera should record at its maximum of 500000 frames per second to obtain a mere 15 data points for the worst-case test of $30 \mu \mathrm{s}$. Recording at this rate reduces the image size to $128 \times 16$ pixels. The low resolution forced averaging of the data over an area approximately equal to a strain gauge. Slower tests were recorded at lower frame rates and increased image dimensions to reduce the noise on the DIC results. The specimens were recorded such that the gauge width at their centre spans the smaller dimension of the camera resolution. Figure 5 shows some recorded frames at a resolution of $128 \times 32$ pixels. DIC calibration was performed to correct for lens distortions.

If 50 data points are taken as a minimum for a stress-strain curve, it means the test duration should be above $100 \mu$ s for the DIC acquisition. For a material with a strain to failure of $1 \%$ this duration limits the maximum strain rate to $100 \mathrm{~s}^{-1}$. The acquisition using strain gauges is not limited by data storage, but rather by amplification. As mentioned before, it is the bandwidth of the signal conditioner at $35 \mathrm{kHz}$ which limits the maximum strain rate.

\subsection{Specimens}

It was chosen to operate the test bench only up to $15 \mathrm{~m} \mathrm{~s}^{-1}$. Assuming the gauge section of the specimens is the only part that deforms, and that this deformation is perfectly elastic, the strain $\varepsilon$ can be calculated by dividing the change in length $\Delta l$ by the gauge section length $l_{g}$. The change in length is then equal to the machine displacement $d$ :

$$
\varepsilon=\frac{\Delta l}{l_{g}}=\frac{d}{l_{g}}
$$


Using equation (1), a theoretical strain rate can be calculated by dividing the rate of displacement, or velocity $v$, with the gauge length:

$$
\dot{\varepsilon}=\frac{v}{l_{g}}
$$

Using equation (2), a gauge length of $75 \mathrm{~mm}$ is theoretically needed to reach a strain rate of $200 \mathrm{~s}^{-1}$ at this velocity. To compensate for the possible effects of grip slippage and bench deformation, a gauge length of $50 \mathrm{~mm}$ was chosen. To approximately reach the strain rates mentioned in the beginning of section 2 , the machine was actuated at $0.0001,0.005,0.05,0.5,5$ and $15 \mathrm{~m} \mathrm{~s}^{-1}$.

To enforce failure away from the clamps, a dumbbell specimen shape was chosen, in contrast to the advise of the test standard for quasi-static tensile testing of composites [1]. This method was effective here, because the investigated lay-ups were pure 90 UD and a plain weave. It would not work for 0 UD specimens, because the relatively low transverse strength results in longitudinal matrix cracking before failure. A shape with cut-outs of a single curvature was used and hence these specimens do not have a gauge section with straight edges. The shape was based on research for fatigue of woven composite specimens [5] and scaled to match the aforementioned strain rate requirements and fit the entire clamping area of the grips. The target dimensions are given in figure 2 Specimens are cut using a waterjet, instrumented and then conditioned according to manufacturer recommendations. Table 1 shows the average dimensions per material. These dimensions lead to a stress concentration factor of about 1.033, determined using a finite-element (FE) simulation, which was applied to all results.

Table 1. Specimen dimensions after waterjet cutting, $L, W_{c}, W_{g}$ and $H$ stand for length, width at clamp, width at centre of gauge and thickness, respectively, see also figure 2

\begin{tabular}{|c|c|c|c|c|c|c|}
\hline & \multicolumn{2}{|c|}{$\mathrm{C} / \mathrm{E}[90]_{8}$} & \multicolumn{2}{|c|}{$\mathrm{C} / \mathrm{E}[\#(0 / 90)]_{2 s}$} & \multicolumn{2}{|c|}{ G/PA-6 $[90]_{8}$} \\
\hline & $\begin{array}{l}\text { Avg } \\
{[\mathrm{mm}]}\end{array}$ & $\begin{array}{l}\text { C.V. } \\
{[\%]}\end{array}$ & $\begin{array}{l}\text { Avg } \\
{[\mathrm{mm}]}\end{array}$ & $\begin{array}{c}\text { C.V. } \\
{[\%]}\end{array}$ & $\begin{array}{l}\text { Avg } \\
{[\mathrm{mm}]}\end{array}$ & $\begin{array}{c}\text { C.V. } \\
{[\%]}\end{array}$ \\
\hline$L:$ & 93.40 & 0.13 & 93.93 & 0.03 & 93.65 & 0.06 \\
\hline$W_{c}:$ & 20.09 & 0.18 & 19.97 & 0.08 & 20.03 & 0.17 \\
\hline$W_{g}:$ & 15.51 & 0.49 & 15.49 & 0.66 & 15.41 & 0.28 \\
\hline$H:$ & 2.35 & 6.78 & 2.04 & 0.88 & 1.87 & 2.31 \\
\hline
\end{tabular}

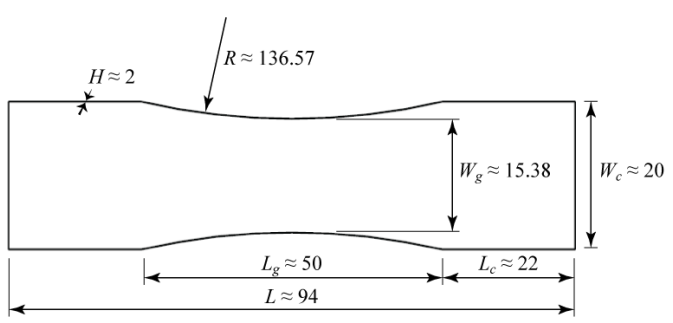

Figure 2. Dogbone specimen shape with target dimensions, scaled from [5]. 


\subsection{Raw results}

Figure 3 contains typical raw results of a $5 \mathrm{~m} \mathrm{~s}^{-1}$ test on $\mathrm{C} / \mathrm{E}[\#(0 / 90)]_{2 s}$. The black parts of the curves indicate what has been selected to belong to the actual test, the red parts are either during the piston speed-up phase or after specimen failure. The initial curved part of the graphs indicates a portion of the test where the bottom grip is still accelerating.

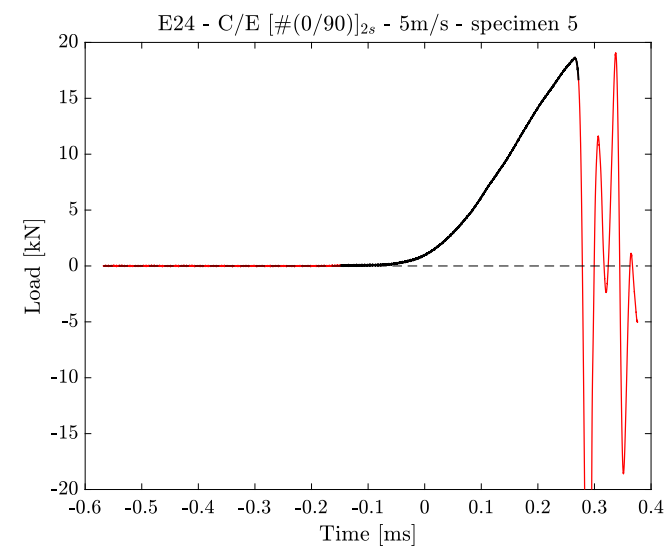

a. Force versus time.

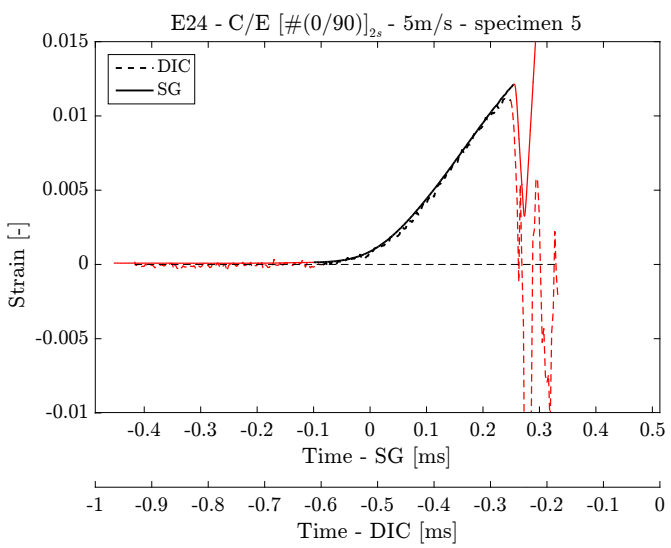

b. Strain versus time.

Figure 3. Typical raw results for $\mathrm{C} / \mathrm{E}[\#(0 / 90)]_{2 s}$ tested at $5 \mathrm{~m} \mathrm{~s}^{-1}$.

The DIC strain shows more noise than the gauge strain. The low resolution of the footage makes that there are only few strain values available per frame, see figure 4 for a visualization of the DIC digestion. This causes the influence of the error of one data point on the average outcome to be rather large. However, the two different strain signals overlap, so the data appears good enough to characterise material properties.

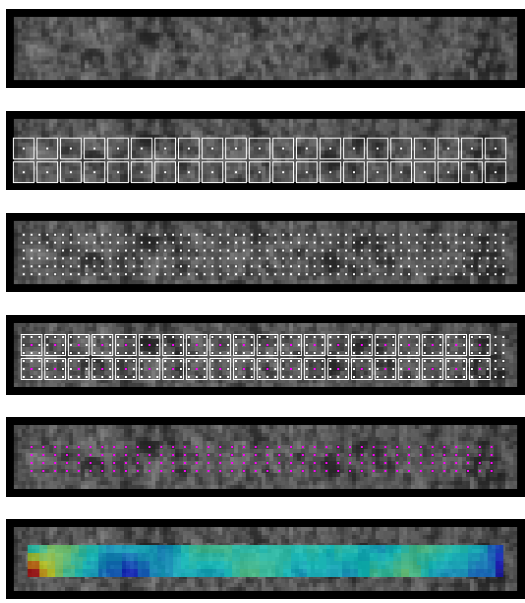

a. A raw frame of $128 \times 16$ pixels.

b. Non-overlapping $5 \times 5$ subsets are shown.

c. The displacement points with a step size of 2 .

d. Choice of strain calculation windows.

e. All available strain points.

f. The resulting strain field.

Figure 4. Illustration of the DIC digestion of a $15 \mathrm{~m} \mathrm{~s}^{-1}$ test recorded at $128 \times 16$ pixels. A subset size of 5 and a step size of 2 were chosen. The zero-normalized sum of square differences correlation algorithm with subsequent cubic interpolation is used to find the displacements at sub-pixel accuracy. The strain is calculated using $3 \times 3$ windows in which linear interpolation is applied. 


\subsection{Strain rate}

The strain rate of a test was approximated by a linear regression on the strain versus time signal, at an interval between strain values of 0.003 and 0.005 , where the strain rate has reached more or less a plateau. This way, a rate of $45.4 \mathrm{~s}^{-1}$ was obtained for the gauge data and $46.6 \mathrm{~s}^{-1}$ for the optical data.

One could also calculate the theoretical strain rate, which is typically taken as the actuation velocity divided by the gauge length [11, 17, see also equation (2). For a $5 \mathrm{~m} \mathrm{~s}^{-1}$ test of a specimen with a gauge length of $50 \mathrm{~mm}$, the theoretical strain rate amounts to $100 \mathrm{~s}^{-1}$.

Both measured strain rate values are lower than the theoretical one, which is also seen in literature [8. The specimens had a gauge length of $50 \mathrm{~mm}$ and were failing at strains of about 0.01 , so only $0.25 \mathrm{~mm}$ of bench deformation or slip is needed to reduce the strain rate to half the theoretical value.

Based on the test data at other speeds, the ratio of actual rate to the theoretical one increases slightly with increasing velocity. The maximum velocity of the test bench equals $20 \mathrm{~m} \mathrm{~s}^{-1}$, resulting in a theoretical rate of $400 \mathrm{~s}^{-1}$ with the current specimen dimensions, based on equation (2). The actual rate is thus limited to about $200 \mathrm{~s}^{-1}$.

\subsection{Load cell ringing}

Ringing of the load cell can be seen in the load signal after failure (figure 3a). A fast Fourier transform was used to investigate this signal. The lowest peak frequency was found to lie at $10.5 \mathrm{kHz}$ and can be taken as the natural frequency of the load measurement assembly [17. If the test duration approaches the period of this oscillation, the measured load value no longer represents the actual load experienced by the specimen, but is rather comprised of an apparently oscillating value. It is explained in section 3.2 .4 that, for results completely free of ringing, the strain rate is limited to values below about $10 \mathrm{~s}^{-1}$.

\section{Results and discussion}

To get from the individual data streams to stress-strain data, the time axes need to be synchronized.

\subsection{Synchronization}

Looking at the raw data curves of figure 3 , one might be inclined to manually align the datasets, as was done by Hufner and Hill [8]. Such an approach, however, does not lead to accurate synchronization for the higher rates, because neither the moment of failure, nor the initial loading can be used for this purpose. Aligning the data at failure is not possible because (1) the strain gauge or DIC pattern detach from the specimen before failure (figure 3b) and (2) the load drop after the specimen breaks is no longer sharp (figure 3a), because the load cell has finite inertia. Also, the 
recorded footage does not clearly show a failure point, but rather a progressing crack, see figure 5 . Aligning on the initial part of the test is not accurate due to an initial delay between stress and strain. The strain increases before the stress does, because strain is measured in the middle of the specimen and load at the fixed end. Fitting the initial parts of the raw data thus implies a small shift backward of the strain data compared to reality which is incorrect.

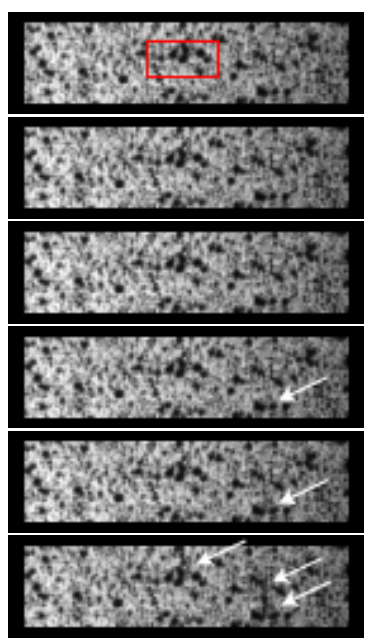

a. The first frame and strain calculation zone.

b. The third to last frame.

c. The second to last frame.

d. The last frame: failure occurs.

e. The first frame after failure.

f. The second frame after failure.

Figure 5. High speed footage of $\mathrm{C} / \mathrm{E}[\#(0 / 90)]_{2 s}$ tested at $15 \mathrm{~m} \mathrm{~s}^{-1}$. The red rectangle indicates the zone of strain calculation, the white arrows indicate show the position of cracks. The capture is done at 360000 frames per second, the resolution is $128 \times 32$ pixels.

\subsubsection{Acquisition delays}

To automatically synchronize stress with strain, the delay of each component in the set-up needs to be known. Figure 1 shows the delay on the signals between each element of the test set-up.

The resistance of the strain gauge and the charge in the load cell are assumed to change without delay with strain/load. Signal travel delays in the wires are also neglected.

The charge amplifier had a delay of $1.87 \mu$ s between charge input and voltage output. The delay between input and amplified output of the signal conditioner was measured to be $2.83 \mu \mathrm{s}$. The trigger delay on the oscilloscope equalled $516 \pm 1 \mu \mathrm{s}$. To cover for the uncertainty on the trigger, this signal was measured again by the oscilloscope.

A trigger pulse of $50 \mathrm{~ns}$ or greater is needed to trigger the camera [15, so a delay of that length was assumed for the start of the recording. At the highest rate of $500000 \mathrm{fps}$, a maximum delay of the timebase of the camera of $2 \mu$ s can occur, because the trigger signal is never received at the exact moment a frame is recorded. To cover for this variable delay, the synchronisation signal of the camera was recorded by the oscilloscope. The $160 \mathrm{~ns}$ delay on this signal [15] was also taken into account.

The remaining uncertainty on the synchronization is estimated to lie below $0.1 \mu \mathrm{s}$. For clarity, 
all delays are summarized in table 2.

Table 2. Summary of acquisition equipment, relevant settings and in- and output delays.

\begin{tabular}{|c|c|c|c|c|}
\hline Equipment & Purpose & $\begin{array}{l}\text { Relevant settings } \\
\text { at highest speed }\end{array}$ & $\begin{array}{l}\text { Input delay } \\
{[\mu \mathrm{s}]}\end{array}$ & $\begin{array}{c}\text { Output delay } \\
{[\mu \mathrm{s}]}\end{array}$ \\
\hline Kistler 5011B & $\begin{array}{l}\text { Charge } \\
\text { amplifier }\end{array}$ & $\begin{array}{l}\text { Short Time Constant, } \\
\text { wideband }\end{array}$ & $-a$ & 1.87 \\
\hline Vishay 2100 & $\begin{array}{c}\text { Signal } \\
\text { conditioner }\end{array}$ & $\begin{array}{l}\text { Minimal gain, } \\
\text { high bandpass mode }\end{array}$ & $-{ }^{a}$ & 2.83 \\
\hline HBM GN412 & $\begin{array}{c}\text { Digital } \\
\text { oscilloscope }\end{array}$ & $\begin{array}{l}100 \text { MSamples s }^{-1}, \\
\text { wideband }\end{array}$ & $<1 \times 10^{-5}$ & $516 \pm 1^{b}$ \\
\hline Photron SA4 & $\begin{array}{l}\text { High-speed } \\
\text { camera }\end{array}$ & $\begin{array}{c}500000 \mathrm{fps} \\
\text { at } 128 \times 16 \text { pixels }\end{array}$ & $0.05+1 \pm 1$ & $0.16^{c}$ \\
\hline \multicolumn{5}{|c|}{${ }^{a}$ Assumed absent } \\
\hline \multicolumn{5}{|c|}{${ }^{b}$ Delay on the trigger output } \\
\hline
\end{tabular}

\subsubsection{Effect on Young's modulus}

Errors of the order of a microsecond can have significant effects at high rates. An example is shown in figure 6, where the strain rate is approximately $45 \mathrm{~s}^{-1}$. The figure shows five stressstrain curves: one at 'Best possible sync,' correcting for all delays in the acquisition sequence, as mentioned in section 3.1.1. The other curves were produced by either delaying the stress or the strain signal. The Young's modulus was calculated by linear fitting to the stress-strain curve in the strain region from 0.1 to $0.3 \%$, as is recommended by the quasi-static test standard [1]. The resulting values are given in the legend of the figure. In the depicted case, the test lasted about $300 \mu \mathrm{s}$, and every $10 \mu \mathrm{s}$ of synchronization error results in a difference of about $5 \mathrm{GPa}$ on the resulting modulus. A typical well-conducted quasi-static tensile test series on composite materials can be expected to show a variation of about $2 \%$ on the Young's modulus [1. The example shows that if the added uncertainty on the modulus measurement due to synchronization is to remain below $1 \%$, the individual data streams should be synchronized to within $1 \mu \mathrm{s}$ in this case. This requirement becomes more stringent when tests are conducted at higher strain rates or on materials with a smaller strain to failure.

Note from the curves in figure 6 that a wrong delay in the stress- or strain data can make the curve show apparent plasticity if the stress is delayed with respect to strain. Alternatively, a too large delay on strain can make the curve shows apparent stiffening. Hence, a sudden appearance of these effects at higher rates probably indicates inaccurate synchronization instead of a rate effect. 


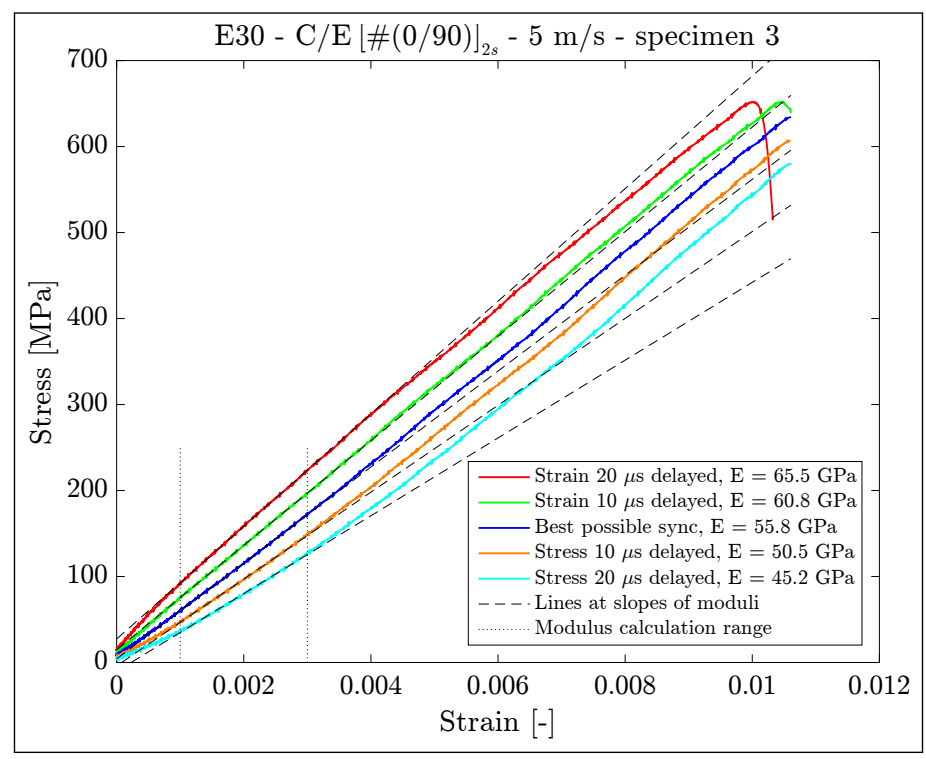

Figure 6. The influence of $10 \mu \mathrm{s}$ steps of synchronisation error between stress and strain on the stressstrain diagram of a $5 \mathrm{~m} \mathrm{~s}^{-1}$ test on $\mathrm{C} / \mathrm{E}[\#(0 / 90)]_{2 s}$. The dashed lines indicate the linear curve fits to calculate the Young's moduli, the vertical dotted lines show the strain range which is used to fit to, as advised by the test standard [1. The calculated values are given in the legend.

\subsection{Physical delay and stress waves}

Strain or stress only propagates inside a material at a finite wave speed. The strain was measured in the centre of the specimen, while the load was measured above the fixed clamp. Hence, the initial load increase occured some time after the strain started to rise.

\subsubsection{Finite-element model of dynamic tensile test}

To investigate how dynamic effects influence the test results without uncertainty about the measurement delays, a 3D FE analysis was carried out. The geometry of the model is shown to the right in figure 7 and modelled after the load train of the dynamic tension set-up depicted to the left in the figure. All parts except the specimen were given the linear elastic properties of steel. The specimen was given a density and a linear elastic material model for which the values are given in table 3. All parts were connected by a tie condition on the mating surfaces. The top of the load cell was restricted in vertical motion. A smooth displacement ramp was applied to the bottom surface of the bottom grip support block using a sinusoidal formulation with different frequencies to match the different test speeds. The model was discretized into 34952 reduced-integration hexahedral elements. An explicit solver was used to run the model, using the default values for bulk damping. Stress and strain were computed from the simulation results in a similar fashion as was done during the experiments. Load was computed by integrating the stress on the midplane of the load cell, see the red line in figure 7, and converted to nominal true stress by dividing by the instantaneous cross-section. The strain was extracted by computing the average value on a surface the size of a strain gauge, see also the blue area in figure 7 . 

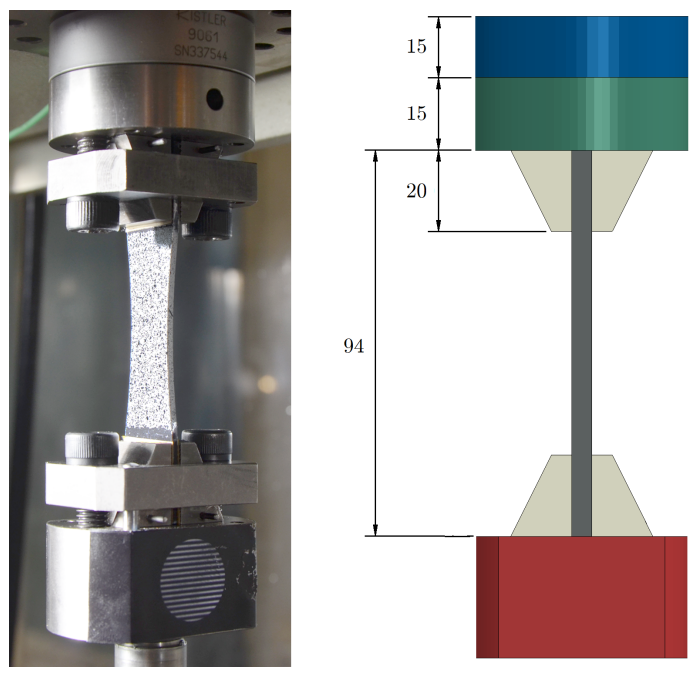

Load cell

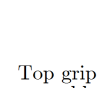

Top grip

assembly

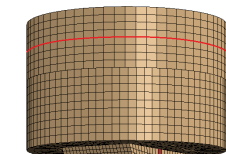

Specimen
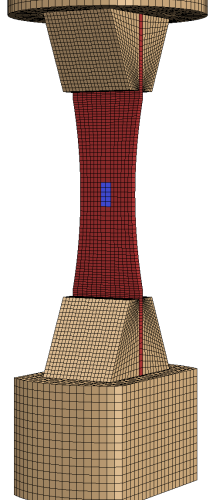

Figure 7. A picture of the set-up (left), dimensions of the dynamic tensile test set-up in mm (middle), and the meshed FE model (right). The red line and blue area in the model show the force- and strain calculation surface areas, respectively.

Table 3. Ply properties used for the different finite element models, to be rotated for use in the FE-model to match the required lay-ups.

\begin{tabular}{ccccc}
\hline Property & Unit & C/E $0^{\circ} \mathrm{UD}$ & $\mathrm{C} / \mathrm{E}[\#(0 / 90)]$ & $\mathrm{G} / \mathrm{PA}-60^{\circ} \mathrm{UD}$ \\
\hline$\rho$ & $\mathrm{kg} \mathrm{m}^{-3}$ & $1560^{a}$ & $1464^{a}$ & $1730^{a}$ \\
$E_{1}$ & $\mathrm{GPa}$ & $135.45^{b}$ & $60.52^{d}$ & $43.5^{e}$ \\
$E_{2}$ & $\mathrm{GPa}$ & $10.76^{b}$ & $59.92^{d}$ & $8.96^{e}$ \\
$E_{3}$ & $\mathrm{GPa}$ & $10.92^{b}$ & $7.47^{d}$ & $8.96^{f}$ \\
$\nu_{12}$ & - & $0.31^{c}$ & $0.07^{d}$ & $0.37^{e}$ \\
$\nu_{13}$ & - & $0.31^{c}$ & $0.46^{d}$ & $0.37^{f}$ \\
$\nu_{23}$ & - & $0.40^{c}$ & $0.46^{d}$ & $0.37^{f}$ \\
$G_{12}$ & $\mathrm{GPa}$ & $5.4^{b}$ & $3.44^{d}$ & $3.14^{e}$ \\
$G_{13}$ & $\mathrm{GPa}$ & $5.4^{b}$ & $2.53^{d}$ & $3.14^{f}$ \\
$G_{23}$ & $\mathrm{GPa}$ & $3.23^{b}$ & $2.58^{d}$ & $1.81^{f}$ \\
\hline
\end{tabular}

Sources: $\quad a$ : Measured using Archimedes method

$b$ : Contact ultrasonics, method explained in [14]

$c: 65 \%$ volume fraction yarn properties [18]

$d$ : Nested MESI finite element model [18]

$e$ : Values from quasi-static tensile tests

$f:$ Estimates 


\subsubsection{Strain acquisition bandwidth}

First, the FE-model was used to evaluate whether the bandwidth of $35 \mathrm{kHz}$ of the signal conditioner used is good enough. Figure 8 contains the results of a simulation of a test at a strain rate of about $300 \mathrm{~s}^{-1}$. The figure also shows strain results filtered by a second order Butterworth filter with several cut-off frequencies to simulate the effect of a limited bandwidth on the obtained strain curve. Only the curve at a $75 \mathrm{kHz}$ bandwidth can reasonably follow the actual strain variation. The SAE requirement for an acquisition bandwidth of $83.3 \mathrm{kHz}$ for a $200 \mathrm{~s}^{-1}$ test seems, therefore, more appropriate than the ISO 26203-2 lower limit of $20 \mathrm{kHz}$. The signal conditioner of the current research had a bandwidth of only $35 \mathrm{kHz}$. Applying the SAE requirement mentioned in the introduction in reverse using the conditioner bandwidth results in a minimum test duration of $71.4 \mu \mathrm{s}$, or about $140 \mathrm{~s}^{-1}$ for a material failing at $1 \%$ strain. At increasingly high values above that rate, the strain signal will appear more and more filtered.

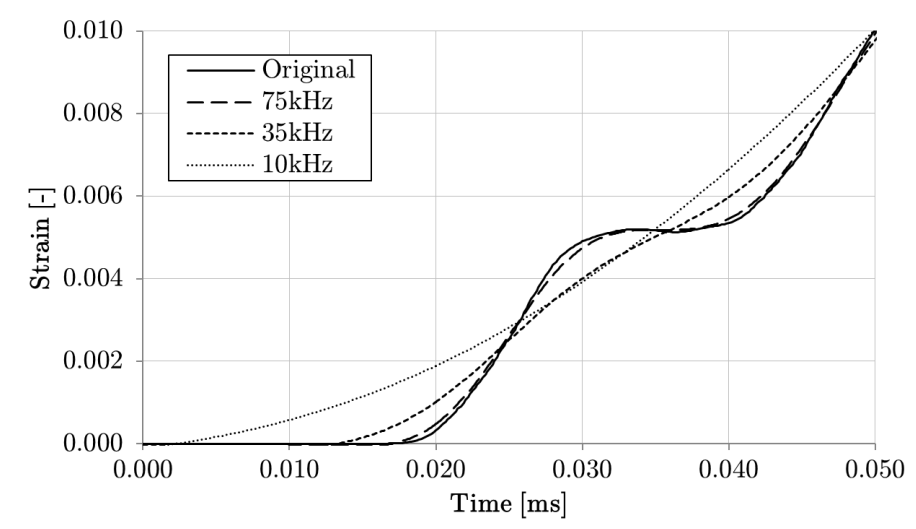

Figure 8. Influence of strain acquisition bandwidth on the obtained curve for a simulated $300 \mathrm{~s}^{-1}$ test on $\mathrm{C} / \mathrm{E}[\#(0 / 90)]_{2 s}$.

\subsubsection{Delaying the strain output}

The delayed increase of the force value with respect to the strain becomes apparent in the results of simulating higher speed tests, compare the red and blue curves in the insert in figure 9. This figure shows that it is incorrect to align load and strain on the initial rise. The original combination (blue curve) retrieves the input Young's modulus (dashed line), while delaying the strain to make it rise when the load does, results in a mismatch (solid black curve). Delaying the strain assumes that information only travels from the loaded end towards the load cell, while in reality the stress waves move in both directions to establish an equilibrium within the load train. Therefore, the stress-strain relation holds in an average sense: the stress waves might show up in the results, but the curves can still be useful to conclude on the overall stress-strain behaviour.

\subsubsection{Influence of load cell ringing on the results}

The load cell ringing frequency in the model amounts to about $57 \mathrm{kHz}$, higher than is found in the real tests $(10.5 \mathrm{kHz})$. This difference is a result of the simplified geometry used in the model, 


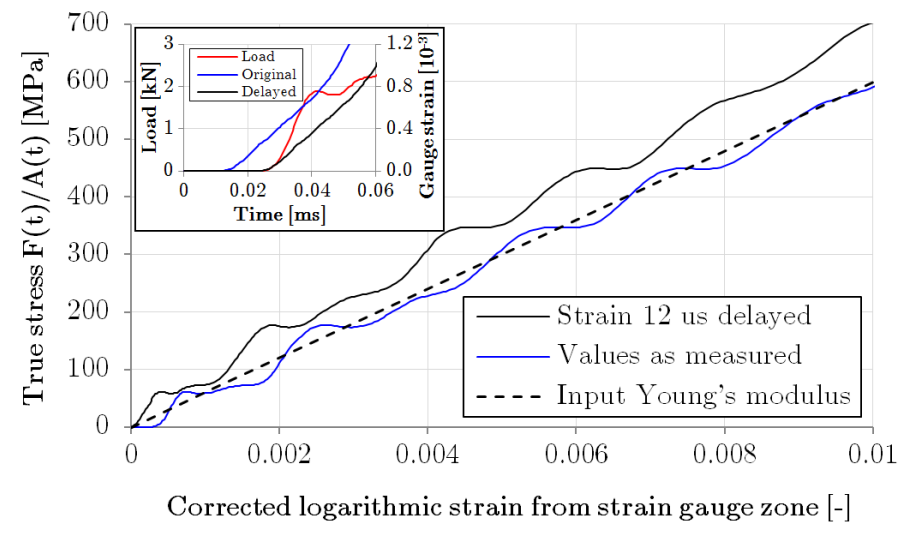

Figure 9. Influence of delaying the strain signal on the stress-strain behaviour of a $5 \mathrm{~m} \mathrm{~s}^{-1}$ test simulated on a $\mathrm{C} / \mathrm{E}[\#(0 / 90)]_{2 s}$ specimen.

especially the absence of the grip clamping structure. However, conclusions can be drawn from the model results. Figure 10 shows simulated force-strain curves obtained at different speeds. At $5 \mathrm{~m} \mathrm{~s}^{-1}$, the oscillation is already clearly visible, although the average behaviour coincides with a linear-elastic response, see also figure 9 The force at higher speeds is no longer usable. The simulation at $5 \mathrm{~m} \mathrm{~s}^{-1}$ has a duration of $160 \mu$ s until $1 \%$ strain, while the oscillation period of the load cell equals about $17.5 \mu \mathrm{s}$. Judging from this result, the authors conclude that the test duration to failure should last ten times the period of load cell oscillation or more. Based on the natural frequency of $10.5 \mathrm{kHz}$, this limits the strain rate to $10.5 \mathrm{~s}^{-1}$ for materials that show a $1 \%$ strain to failure. Above this limit, the results are not immediately completely invalid, though one should realise that the oscillations in the load data are not related to the stress in the specimen. As the load measurement system has already been optimized to reach a high natural frequency, a different solution is advised to test at higher rates. Possible candidates are using a 'dynamometer section' [10] or ultra-high speed image acquisition [16] to measure the load on-specimen.

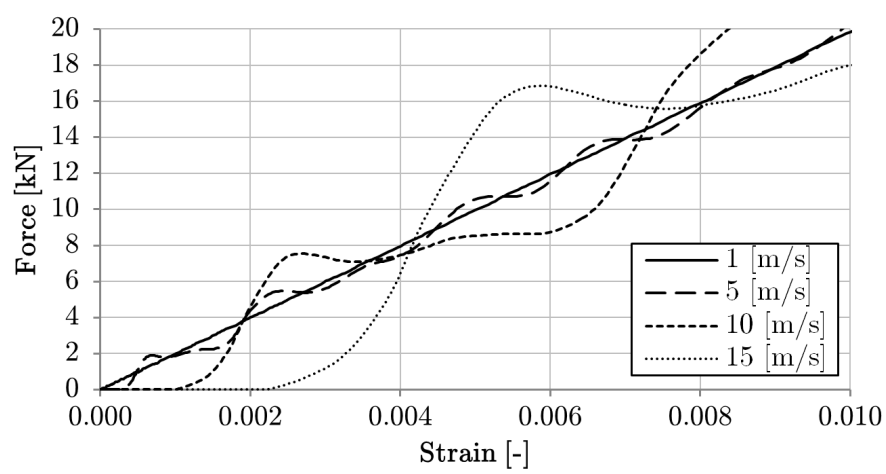

Figure 10. Stress-strain behaviour from simulations at up to $15 \mathrm{~m} \mathrm{~s}^{-1}$, strongly influenced by cell ringing at the higher speeds. 


\subsubsection{Equilibrium}

Even when using a different manner of measuring load, the maximum strain rate in the current configuration is limited to an upper bound. At high speeds, the strain no longer has the time to evenly distribute over the specimen. The effects become noticeable above a velocity of $5 \mathrm{~m} \mathrm{~s}^{-1}$, see figure 11. At that speed, the load pulse duration to $0.5 \mathrm{~mm}$ displacement equals $100 \mu \mathrm{s}$. This is slightly more than the time needed for a pulse to travel three times back and forth from end to end in the $\mathrm{C} / \mathrm{E}[\#(0 / 90)]_{2 s}$ specimen. Apparently, this time is just sufficient for an approximate equilibrium to exist within the specimen. This also matches the condition posed by Xiao based on established research on SHPB testing [21]. This condition leads to an upper bound in strain rate $\dot{\varepsilon}^{\text {max }}$ that depends on the specimen length $L$, the failure strain $\varepsilon_{f}$ and the speed of sound $c$ in the tested material. Assuming a linear increase of strain with time, i.e. a constant strain rate, the relationship becomes as follows.

$$
\dot{\varepsilon}^{\max }=\frac{\varepsilon_{f} c}{6 L}
$$

For the $\mathrm{C} / \mathrm{E}[\#(0 / 90)]_{2 s}$ specimen of $94 \mathrm{~mm}$ in length, this amounts to a maximum attainable strain rate of $107 \mathrm{~s}^{-1}$. To reach higher rates, apart from the necessity to apply a different method to measure load, specimens should be shorter. However, shorter dumbbell specimens have the drawback that the stress concentration is higher. Moreover, below a certain length, added nonuniformity will appear in the stress distribution due to the proximity of the clamps. The $90^{\circ}$-UD material shows a slower wave propagation, limiting the strain rate to $30 \mathrm{~s}^{-1}$ for $\mathrm{C} / \mathrm{E}$ and $50 \mathrm{~s}^{-1}$ for G/PA-6.

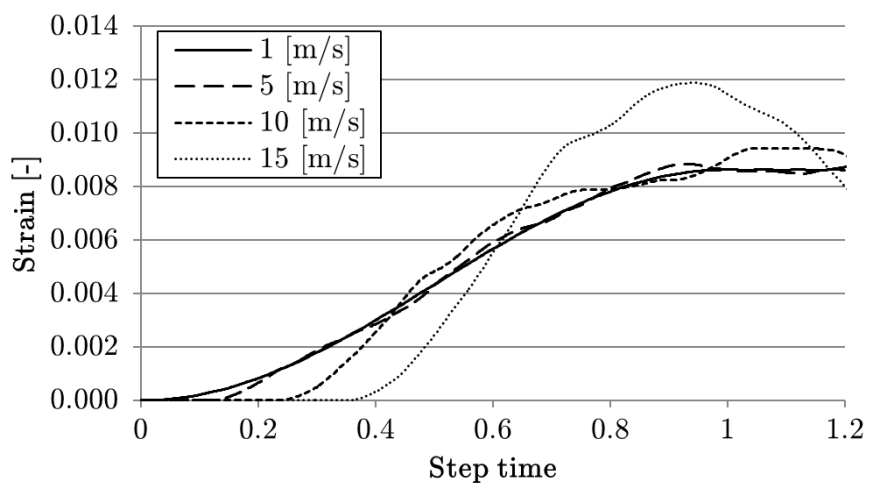

Figure 11. Strain versus step time ( $t=1$ at $0.5 \mathrm{~mm}$ displacement, here held constant afterwards) at up to $15 \mathrm{~m} \mathrm{~s}^{-1}$. Above $5 \mathrm{~m} \mathrm{~s}^{-1}$, the strain visibly overshoots the equilibrium value.

\subsubsection{Summary of strain rate limits}

All the limiting factors of the current test set-up and resulting maximum attainable strain rates are summarised in table 4 . Already at $10 \mathrm{~s}^{-1}$ the results will start to deviate from reality. Up to about $50 \mathrm{~s}^{-1}$ the influence is still minor, but above that rate the results are invalidated by a combination of the effects mentioned above (see also figure 12b. The test set-up needs to be 
changed for it to produce accurate results at higher rates: load should be measured on-specimen, the specimen should be shorter and slightly better acquisition equipment is needed. The following

Table 4. Summary of strain rate limits for the current test set-up.

\begin{tabular}{|c|c|c|c|c|c|}
\hline$\dot{\varepsilon} \operatorname{limit}\left[\mathrm{s}^{-1}\right]$ & 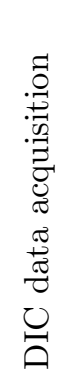 & 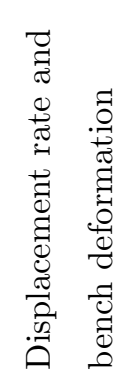 & 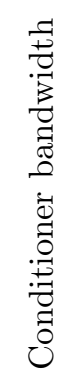 & 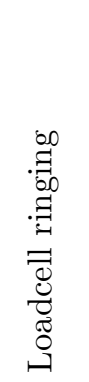 & 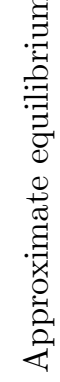 \\
\hline $\mathrm{C} / \mathrm{E}[90]_{8}$ & 60 & 200 & 84 & 6.4 & 30 \\
\hline G/PA-6 $[90]_{8}$ & 100 & 200 & 140 & 10.5 & 50 \\
\hline $\mathrm{C} / \mathrm{E}[\#(0 / 90)]_{2 s}$ & 100 & 200 & 140 & 10.5 & 107 \\
\hline
\end{tabular}

list contains which values need to be measured to determine the resulting limit on strain-rate for each of the five criteria for a specific combination of tested material and test equipment. The test duration can be approximated by dividing the strain to failure by the strain rate.

- DIC data acquisition:

- Input:

* Requested minimal amount of data points per test curve

* Maximum acquisition rate

* Strain to failure

- Criterion: the test duration >amount of data points divided by the acquisition rate

- Displacement rate and bench deformation:

- Input:

* Maximum speed of the test bench

* Specimen gauge length

* Factor between actual and theoretical strain-rate (0.5 is a conservative assumption)

- Criterion: the maximum rate is equation 2 multiplied by the factor

- Signal conditioner bandwidth:

- Input:

* Signal conditioner bandwidth

* Strain to failure 
- Criterion: the cut-off frequency $>10 \mathrm{x}$ the reciprocal of $4 \mathrm{x}$ the test duration

- Load cell ringing:

- Input:

* Ringing frequency

* Strain to failure

- Criterion: the test duration $>10 \mathrm{x}$ the period of load cell ringing

- Approximate equilibrium within the specimen:

- Input:

* Strain to failure

* Speed of sound in material test direction

* Specimen length

- Criterion: use equation (3) to find the maximum strain rate

\subsection{Final test results}

Figure 12 contains an overview of stress-strain data of $\mathrm{C} / \mathrm{E}[\#(0 / 90)]_{2 s}$ tested at different speeds. The curves in figure $12 \mathrm{a}$ were obtained by synchronization on the initial parts of stress and strain, those in figure $12 \mathrm{~b}$ result from considering all the acquisition delays. Only one curve has been included for each of the four lowest rates because they fall on one line. This indicates that the general behaviour is rate-insensitive at those speeds. There is no noticeable difference between the two figures for these curves. The data at the two highest rates, however, does show the influence of synchronization. The strain rate of $148 \mathrm{~s}^{-1}$ surpasses most of the limits listed in table 4. The waviness in the red stress-strain result is attributed to a combination of load cell ringing and the absence of equilibrium in the specimen. The result cannot be used and only one curve was added for this reason. Five stress-strain curves are included in blue belonging to the tests with a target speed of $5 \mathrm{~m} \mathrm{~s}^{-1}$. The average strain rate of these tests lies at $45.8 \mathrm{~s}^{-1}$ : below most limits of table 4. While the curves nicely fall on top of those at lower rates in figure $12 \mathrm{~b}$, in figure $12 \mathrm{a}$ they appear above the low-rate results. This shows that a wrong synchronization method could cause an apparent strain rate effect to appear in the test results. In this case, the average Young's modulus would be falsely increased from 58.2 GPa to $64.4 \mathrm{GPa}$. The consideration of all delays, however, results in a reliable set of stress-strain curves including the ones at higher rates. Correct synchronization is thus key in obtaining reliable test results at high rates. It can be concluded based on this data that $\mathrm{C} / \mathrm{E}[\#(0 / 90)]_{2 s}$ shows no significant rate effect until at least $45.8 \mathrm{~s}^{-1}$.

Taking all the acquisition delays into account, the stress-strain curves of $\mathrm{C} / \mathrm{E}[90]_{8}$ at six different rates become as is displayed in figure $13 \mathrm{a}$. The average stress-strain curves are calculated 


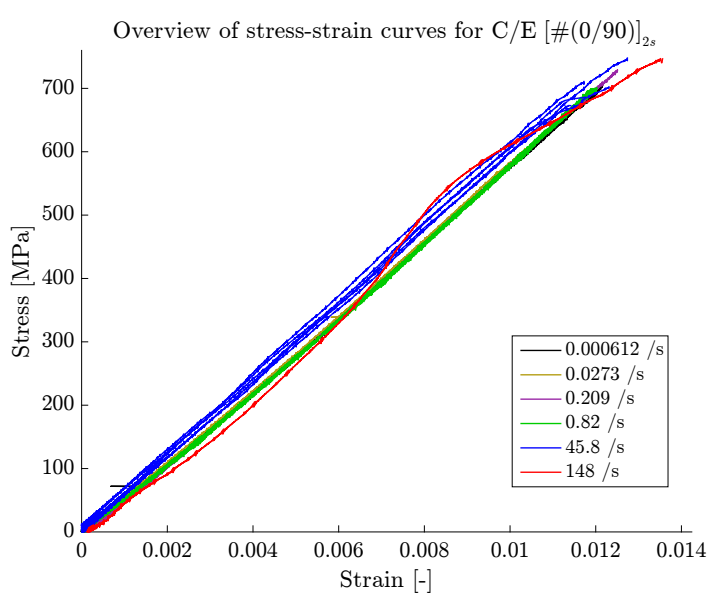

a. Stress-strain curves from synchronization on the initial stress and strain behaviour.

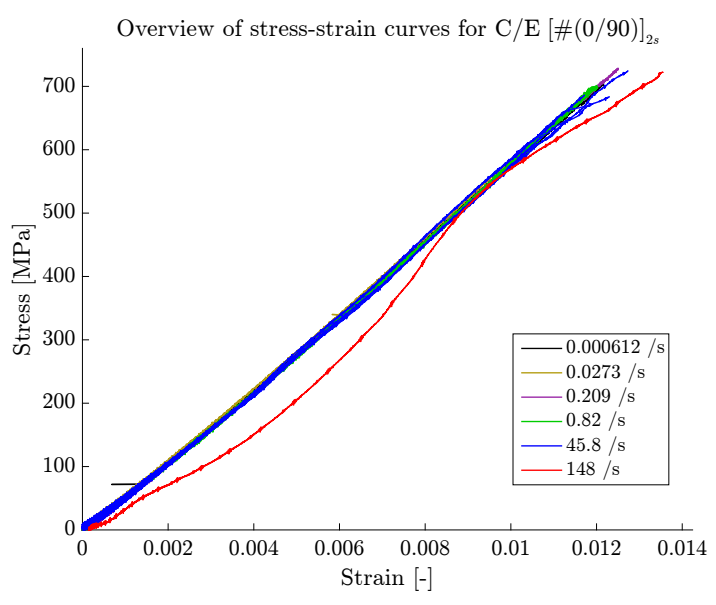

b. Stress-strain curves by taking into account the acquisition delays.

Figure 12. The influence of synchronization on the results of $\mathrm{C} / \mathrm{E}[\#(0 / 90)]_{2 s}$ at different strain rates.

by fitting a fourth-order polynomial to the collective results of each rate. The source curves are shown only for the highest and the lowest rate to keep the figure clear. The fitted curves stop at the average maximum strain measured at that rate. Strain was measured mainly using DIC in the UD test series. The large amount of noise on the strain acquisition is striking, compare the background curves of figure $13 \mathrm{a}$ to the curves in figure 12 . This is not only a result of the low resolution, but also due to the fact that the assumption of strain equilibrium is severely violated for this material at the highest rate. Also, the small failure strain of the $\mathrm{C} / \mathrm{E}$ in transverse direction reduces the signal-to-noise ratio. It is again concluded that the resolution of the used camera is small, although still the general strain trends can be measured. The stress-strain curves of G/PA-6 $[90]_{8}$ at six different rates are shown in figure $13 \mathrm{~b}$. Again, the source curves are shown only for the highest and the lowest rate. Both material systems show a small increase in Young's modulus and failure stress with strain rate. No clear trend can be discerned for the failure strain. 


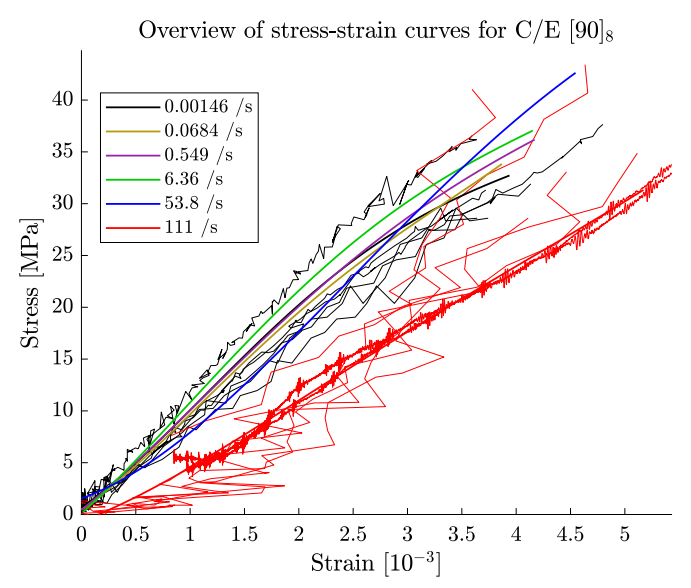

a. Stress versus strain for $\mathrm{C} / \mathrm{E}[90]_{8}$ at six different strain rates. Each curve is an average of at least five results, the original curves are indicated for the lowest and the highest rate.

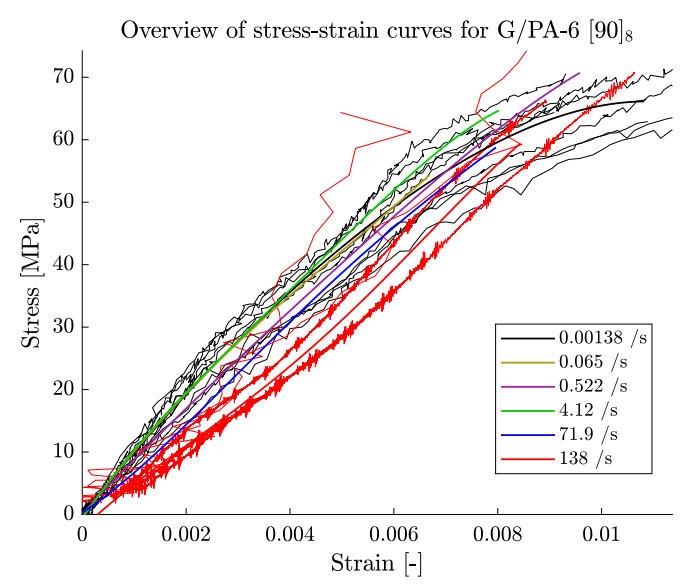

b. Stress versus strain for G/PA-6 $[90]_{8}$ at six different strain rates. Each curve is an average of at least five results, the original curves are indicated for the lowest and the highest rate.

Figure 13. Dynamic tension test results on two transverse UD composites. Please note that the curves for the highest rates are invalid as several limits mentioned in table 4 have been surpassed.

\section{Conclusions}

A hydraulic pulse test bench was used in an attempt to characterise the tensile response of brittle continuous-fibre-reinforced composites up to $200 \mathrm{~s}^{-1}$. Two material systems and lay-ups with a failure strain at or below $1 \%$ were tested. Strain was measured using both strain gauges and digital image correlation.

Synchronization between different measurement systems was found to be essential. A onemicrosecond-delay can influence the computed Young's modulus by $1 \%$ at $45 \mathrm{~s}^{-1}$, or $0.5 \mathrm{GPa}$ in the example shown. Synchronizing on the beginning of the time histories is invalid due to the delay between the initial rise of force and strain resulting from a different measurement location of these quantities. Using the moment of failure is inaccurate because the precise capturing of this moment is hindered by the debonding of strain gauges or DIC paint, and the inertia of the load cell. The only remaining option is to take into account the delay of every element in the chain of data acquisition, which was proven to result in reliable test curves.

Multiple conclusions were drawn regarding the limiting factors on the maximum strain rate using a hydraulic pulse test bench.

1. The combination of specimen length and maximum test bench velocity should allow for the rate to be reached, where it can be assumed that the actual rate equals approximately half the theoretical rate for brittle composites.

2. The data acquisition systems need a bandwidth exceeding ten times the fastest expected 
variation of the input signals, which can be approximated by assuming that the shortest test duration equals one quarter of a sinusoidal period. Data sampling should occur at minimally twice that frequency.

3. Digital image correlation using high speed cameras can be applied to measure strain at high rates. However, the reduction in resolution needed to capture strain at $5 \mathrm{~s}^{-1}$ or higher reduces the acquisition to a single value. Ultra-high speed cameras could be a solution to acquire local rather than only global strain values.

4. The period of the natural frequency of the load measurement assembly should be ten times smaller than the test duration for the results to be completely free of load oscillations. The only way to overcome this hurdle is to measure the load on-specimen. Investigation into the possibility of adhering an instrumented and rate-insensitive metal part to the specimen to act as a load cell is currently underway.

5. An approximate equilibrium should exist within a specimen to obtain an accurate stressstrain curve based on distinct measurement locations for stress and strain. This approximate equilibrium can be assumed to exist when the test duration is larger than three times the time needed for a stress wave to pass back and forth between the ends of the specimen.

All in all, a classic tensile test at high speed seems a suitable method to investigate the rate dependency of brittle composites. Usable full tensile stress-strain curves could be obtained at strain rates ranging from quasi-static up to $50 \mathrm{~s}^{-1}$. The curves show that the $\mathrm{C} / \mathrm{E}[\#(0 / 90)]_{2 s}$ is rate insensitive in the examined range, while both $\mathrm{C} / \mathrm{E}[90]_{8}$ and G/PA-6 $[90]_{8}$ show an increase of Young's modulus and failure stress with strain rate. The applicability of the method at higher rates is the subject of further research, focussing mainly on the load measurement.

\section{Acknowledgements}

This work was supported by the SBO project M3Strength, which fits in the MacroModelMat (M3) research program funded by SIM (Strategic Initiative Materials in Flanders) and VLAIO (Flemish government agency for Innovation and Entrepeneurship). E. Verboven acknowledges the financial support of the Fonds Wetenschappelijk Onderzoek (FWO) with project G0B9515N and M. Kersemans acknowledges the financial support of FWO fellowship 12T5418N. The authors express their gratitude towards Honda R\&D Co., Ltd., Mitsubishi Rayon Co., Ltd., and Ten Cate Advanced Composites B.V. for their generosity in supplying materials for this research. They also wish to thank M. Dolphen and N. Szymański for their extensive aid in the testing programme.

\section{References}

[1] ASTM International. ASTM standard D3039, 2008, "Standard test method for tensile properties of polymer matrix composites". Technical report, West Conshohocken, PA, 2008. 
[2] I. M. Daniel. High strain rate properties of angle-ply composite laminates, part 3 (Final report). Contractor Report 189085, NASA, 1991.

[3] I. M. Daniel. High strain rate properties of off-axis composite laminates, part 2. Contractor Report 189084, NASA, December 1991.

[4] I. M. Daniel. High strain rate properties of unidirectional composites, part 1. Contractor Report 189083, NASA, December 1991.

[5] Ives De Baere, Wim Van Paepegem, Christian Hochard, and Joris Degrieck. On the tension-tension fatigue behaviour of a carbon reinforced thermoplastic part II: evaluation of a dumbbell-shaped specimen. Polymer Testing, 30(6):663-672, 2011. doi:10.1016/j.polymertesting.2011.05.005

[6] ESIS TC 5, Subcommittee on Dynamic Testing at Intermediate Strain Rates. ESIS P7-00: procedure for dynamic tensile tests. Technical report, European Structural Integrity Society, 2000.

[7] Hottinger Baldwin Messtechnik GmbH. HBM GEN series GN412. Data sheet B2627-2.1 en, October 2016.

[8] David R Hufner and Susan I Hill. High strain rate testing and modeling of a woven eglass-vinylester composite in dry and saturated conditions. Journal of Composite Materials, page 0021998316681185, December 2016. ISSN 0021-9983. doi:10.1177/0021998316681185

[9] International Organization for Standardization. ISO 18872:2007(E). Plastics - Determination of tensile properties at high strain rates. Technical report, International Organization for Standardization, 2007.

[10] International Organization for Standardization. ISO 26203-1:2010. Metallic materials - Tensile testing at high strain rates - Part 1: Elastic-bar-type systems. german version DIN EN ISO 26203-1:2010. Technical report, International Organization for Standardization, 2010.

[11] International Organization for Standardization. ISO 26203-2:2011. Metallic materials - Tensile testing at high strain rates - Part 2: servo-hydraulic and other test systems. german version DIN EN ISO 26203-2:2011. Technical report, International Organization for Standardization, 2011.

[12] Kistler Instrumente AG. Kistler 5011B charge amplifier, 2005.

[13] Peter Kuhn, Marina Ploeckl, and Hannes Koerber. Experimental investigation of the failure envelope of unidirectional carbon-epoxy composite under high strain rate transverse and off-axis tensile loading. EPJ Web of Conferences, 94:01040, 2015. ISSN 2100-014X. doi:10.1051/epjconf/20159401040. 
[14] N. Lammens, M. Kersemans, Ives De Baere, and W. Van Paepegem. On the viscoelasto-plastic response of additively manufactured polyamide-12 (PA-12) through selective laser sintering. Polymer Testing, 57:149-155, February 2017. ISSN 01429418. doi $10.1016 /$ j.polymertesting.2016.11.032.

[15] Photron Limited. FASTCAM SA4 hardware manual - revision 1.06E. Technical Report E120120644U, Tokyo, January 2012.

[16] Fabrice Pierron and Michel Grédiac. The Virtual Fields Method. Springer New York, New York, NY, 2012. ISBN 978-1-4614-1823-8 978-1-4614-1824-5. DOI: 10.1007/978-1-4614-1824-5.

[17] SAE International. SAE J2749, 2017, "Surface vehicle recommended practise - high strain rate testing of polymers". Technical report, 400 Commonwealth Drive, Warrendale, PA 15096, July 2017.

[18] R. D. B. Sevenois, D. Garoz, F. A. Gilabert, S. W. F. Spronk, S. Fonteyn, M. Heyndrickx, L. Pyl, D. Van Hemelrijck, J. Degrieck, and W. Van Paepegem. Avoiding interpenetrations and the importance of nesting in analytic geometry construction for representative unit cells of woven composite laminates. Composites Science and Technology, 136:119-132, November 2016. ISSN 0266-3538. doi $10.1016 /$ j.compscitech.2016.10.010

[19] R. L. Sierakowski. Strain rate effects in composites. Applied Mechanics Reviews, 50(12): 741-761, 1997. doi $10.1115 / 1.3101860$.

[20] H. Werner and H. Gese. Zur bedeutung dehnratenabhängiger werkstoffkennwerte in der crashsimulation. In Kennwertermittlung für die Praxis, pages 139-146. WILEY-VCH Verlag GmbH \& Co. KGaA, May 2007.

[21] Xinran Xiao. Dynamic tensile testing of plastic materials. Polymer Testing, 27(2):164-178, April 2008. ISSN 0142-9418. doi:10.1016/j.polymertesting.2007.09.010. 